Pacific Journal of Mathematics

SCHRÖDINGER SEMIGROUPS ON THE SCALE OF SOBOLEV 


\title{
SCHRÖDINGER SEMIGROUPS ON THE SCALE OF SOBOLEV SPACES
}

\section{BARRY SIMON}

\begin{abstract}
We consider the action of semigroups $e^{-t H}$, with $H=-\Delta+V$ on $L^{2}\left(R^{\nu}\right)$, on the scale of Sobolev spaces $\mathscr{H}^{\alpha}$. We show that while $e^{-t H}$ maps $L^{2}=\mathscr{H}^{0}$ to $\mathscr{H}^{2}$ under great generality, there exist bounded $V$ so that, for all $\beta>0, e^{-t H}\left[\mathscr{H}^{\beta}\right]$ is not contained in any $\mathscr{H}^{\alpha}$ with $\alpha>2$.
\end{abstract}

1. Introduction. This note represents a modest contribution to the issue of smoothing properties of Schrödinger semigroups, $e^{-t H}, H=-\Delta$ $+V$ on $L^{2}\left(R^{\nu}\right)[12]$. It has been shown $[3,8,2,11,12]$ under fairly great generality (i.e. assumptions on $V$ ) that $e^{-t H}$ is smoothing on the scale of $L^{p}$ spacses, i.e. $e^{-t H}$ maps $L^{p}$ into any $L^{q}$ with $q \geq p$. Kon [7] asked the question of smoothing properties on the scale of Sobolev spaces $\mathscr{H}^{\alpha}$. Below we will exploit their $L^{q}$ analogs, so we define them: $f \in L^{q}\left(R^{\nu}\right)$ is said to lie in $L_{\alpha}^{q}(\alpha \geq 0)$ if there exists $g \in L^{q}\left(R^{\nu}\right)$ so that $\hat{g}(p)=$ $\left(1+|p|^{2}\right)^{\alpha / 2} \hat{f}(p) \cdot L_{\alpha}^{2} \equiv \mathscr{H}^{\alpha}$. We will also require the spaces $K_{\nu}$ defined initially by Kato [5]: If $\nu=1$,

otherwise

$$
K_{\nu}=\left\{f\left|\sup _{x}\left[\int_{x-1}^{x+1}|f(y)| d y\right]<\infty\right|\right\}
$$

$$
K_{\nu}=\left\{f\left|\lim _{\alpha \downarrow 0}\left[\sup _{x} \int_{|x-y| \leq \alpha} B_{\nu}(x-y)|f(y)| d^{\nu} y\right]=0\right|\right\}
$$

where $B_{\nu}(x)=|x|^{-(\nu-2)}$ if $\nu \geq 3$ and $B_{2}(x)=-\ln |x|$. For any of these spaces $\chi$, we define $\chi_{\text {loc }}=\left\{f \mid f \varphi \in \chi\right.$ for all $\left.\varphi \in C_{0}^{\infty}\left(R^{\nu}\right)\right\}$. We summarize properties of these spaces needed below in an appendix.

Consider for a moment $\nu=3$. It is well known [6] that if $V \in$ $\left(L^{2}+L^{\infty}\right)\left(R^{3}\right)$, then $D(H)=\mathscr{H}^{2}$, and thus obviously $e^{-t H}$ maps $\mathscr{H}^{0}=$ $L^{2}$ to $\mathscr{H}^{2}$. Since there is lots of room between $L^{2}$ and $L^{\infty}$, one might hope that for any $V \in L^{\infty}, L^{2}$ is mapped into some $\mathscr{H}^{\alpha}$ with $\alpha>2$. Our main result in $\S 2$ will be to show there are $V \in L^{\infty}$ with compact support, so that $\operatorname{Ran}\left(e^{-t H}\right)$ is not in any $\mathscr{H}^{\alpha}$ with $\alpha>2$. Indeed, we will prove:

TheOREM 1. Suppose that $V_{+}=\max (V, 0) \in K_{\nu}^{\text {loc }}$ and $V_{-}=$ $\max (-V, 0) \in K_{\nu}$ and that $H e^{-t H} \varphi$ and $e^{-t H} \varphi$ lie in $\mathscr{H}_{\mathrm{loc}}^{\alpha}$ for some $\alpha>2$ and for one $\varphi \geq 0(\varphi \neq 0)$. Then for $\beta=\min (\alpha-2,1), V \in L_{\beta, \mathrm{loc}}^{4 / 3}$. 
The example above will come from the fact that there exist $V \in L^{\infty}$ of compact support but in no $L_{\beta}^{4 / 3}$ with $\beta>0$.

In the above motivating discussion, we obtain mapping onto $\mathscr{H}^{2}$ by using $D(H)=D(-\Delta)$. This is not necessary; $V \in L_{\text {loc }}^{2}$ suffices if we only want local results, for in $\S 3$, we prove

Theorem 2. If $V_{-} \in K_{\nu}, V_{+} \in L_{\mathrm{loc}}^{1}$ and $V \in L_{\mathrm{loc}}^{p}$ on some open set $S$, then $e^{-t H}$ maps $L^{2}$ to functions in $L_{\alpha=2, \text { loc }}^{p}$ on $S$.

Of course, to get additional smoothing, one needs more smoothness on $V$. In $\S 3$, we also prove $\alpha^{\prime}<\alpha$.

THEOREM 3. If $V \in L_{\alpha, \text { loc }}^{\infty}$, then $e^{-t H}$ maps $L^{2}$ to $\mathscr{H}_{\mathrm{loc}}^{\alpha^{\prime}+2}$ for all

We note that Sobolev imbedding theorems imply that $L_{\beta, \text { loc }}^{p} \subset L_{\alpha, \text { loc }}^{\infty}$ if $0<\alpha<\alpha^{\prime} \equiv \beta-\nu p^{-1}$.

The astute reader will note the presence of $L^{p}$ conditions on the potential with $p<2$ in Theorem 1 and $p>2$ in Theorem 3 . One might hope that with more clever arguments one could get away with sharp $L^{2}$ conditions. We do not have enough evidence to call the statement below a conjecture, although it would be pretty if true:

Open question. Is it true that $e^{-t H}$ maps $L^{2}$ to $\mathscr{H}_{\mathrm{loc}}^{\alpha}$ with $\alpha>2$ if and only if $V \in L_{\alpha-2, l o c}^{2}$ ?

We remark that there are earlier results of Hunziker [4] on smooth $V$ 's yielding $e^{-t H}$ mapping the Schwartz space, $\mathscr{S}$, to itself. Indeed, by using the ideas of [10], one can prove that if $D^{\alpha} V \in L^{\infty}$ for all $\alpha$, then $e^{-t H}$ maps $\mathscr{S}$ to itself. From this, it immediately follows that $e^{-t H}$ maps $\mathscr{S}$ to itself.

The counterexample in this paper involves the most regular of elliptic operators with mildest noncontinuity possible, namely, a noncontinuity in the lowest order term. We show that the semigroup for the operator which is normally the most regularizing function of the operator already does not act particularly well on the scale of Sobolev spaces. This illustrates that the Sobolev spaces are not really well suited to the study of partial differential operators with noncontinuous coefficients.

\section{Negative results.}

Proof of Theorem 1. Let $\psi=e^{-t H} \varphi$. By hypothesis, $\psi \in \mathscr{H}_{\mathrm{loc}}^{\alpha}$. Moreover, by general principles (see Cor. B.3.2, Thm. B.7.1 and the proof of 
Lemma B.7.7 in [12]), $\psi$ is continuous and everywhere strictly positive. Since $\psi \in \mathscr{H}^{2} \subset \mathscr{H}^{\alpha}$, we have, by Theorem A.6 that $\psi \in L_{1}^{4}$, and then by Theorem A.5 that $\psi^{-1} \in L_{1}^{4}$. But since $H \psi \in \mathscr{H}_{\mathrm{loc}}^{\alpha}$ by hypothesis and $-\Delta \psi \in \mathscr{H}_{\text {loc }}^{\alpha-2}$ by hypothesis, $V \psi=H \psi+\Delta \psi \in \mathscr{H}_{\text {loc }}^{\alpha-2}$, so by Theorem A.4, $V=\psi^{-1}(V \psi) \in L_{\beta}^{4 / 3}$ where $\beta=\min (\alpha-2,1)$. This completes the proof of Theorem 1 .

REMARKS (1) As noted in the introduction, there exist $V \in L^{\infty}$ with $V \notin L_{\beta, \text { loc }}^{4 / 3}$ for all $\beta>0$. Thus there exist $V \in L^{\infty}$ so $e^{-t H}$ does not map $L^{2}$ to $\mathscr{H}^{\alpha}(\alpha>2)$ for any $s>0$. For if it does, then for $t>s, e^{-t H}$ and $H e^{-t H}=e^{-s H} H e^{-(t-s) H}$ map $L^{2}$ to $\mathscr{H}^{\alpha}$.

(2) It cannot even be true if $V \notin L_{\beta}^{4 / 3}$, that $e^{-t H}\left(t \geq t_{0}\right)$ maps $\mathscr{H}^{\gamma}$ to $\mathscr{H}^{\alpha}$ with $\alpha>0$. For if it did, by applying the Stein interpolation theorem, $e^{-t H}$ would map $\mathscr{H}^{\gamma-\varepsilon}$ to $\mathscr{H}^{\alpha-\varepsilon^{\prime}}$ holomorphically in $s$ for $s$ near $t_{0}$, and so $H e^{-s h}$ would also map $\mathscr{H}^{\gamma-\varepsilon}$ to $\mathscr{H}^{\alpha-\varepsilon^{\prime}}$.

\section{Positive results.}

Proof of Theorem 2. By general principles (Theorem B.1.1 of [22]), $e^{-t H}$ maps $L^{2}$ to $L^{\infty}$, so if $\varphi \in L^{2}, V e^{-t H} \varphi \in L_{\text {loc }}^{p}$ and $H e^{-t H} \varphi \in L^{\infty} \subset$ $L_{\text {loc }}^{p}$, so $-\Delta\left(e^{-t H} \varphi\right)=(H-V) e^{-t H_{\varphi}} \in L_{\text {loc }}^{p}$. Since $e^{-t H} \varphi \in L_{\text {loc }}^{p}$, we have, by Theorem A.8, that $e^{-t H} \varphi \in L_{\alpha=2, \text { loc }}^{p}$

REMARK. It is known (Theorem B.2.1 of [12]) that $(H+c)^{-a}: L^{2} \rightarrow$ $L^{\infty}$ if $a>n / 4$. The above proof shows that if $\tilde{a}>1+n / 4$, then $(H+c)^{-\tilde{a}}$ maps $L^{2}$ to $L_{\alpha=2, \text { loc }}^{p}$.

Proof of Theorem 3. Suppose first $S=R^{\nu}$. By Theorem 2, $f \equiv e^{-t H} \varphi$ $\in L_{2, \text { loc }}^{\infty} \subset L_{2, \text { loc }}^{p}$, all $p<\infty$ (by Theorem A.2). Since $V \in L_{\alpha, \text { loc }}^{\infty}$, it follows by Theorem A.1 that $V f \in L_{\beta, \text { loc }}^{p}$ with $\beta=\min (\alpha, 2), p<\infty$ and so, by a Sobolev estimate, $V f \in L_{\beta, \text { loc }}^{\infty}$, all $\beta^{\prime}<\beta$. Thus, since $H e^{-t H} \varphi \in L_{2, \text { loc }}^{\infty}$, we see that $\Delta f \in L_{\beta^{\prime}, \text { loc }}^{\infty}$, i.e. by Theorem A.8, $f \in L_{\beta^{\prime}+2 \text {,loc }}^{\alpha}$ This completes the proof if $\alpha \leq 2$. If $\alpha>2$, we have $f, H f \in L_{\eta, \text { loc }}^{\infty}$, all $\eta<2$, and we can repeat the argument above to learn $f \in L_{\beta^{\prime} \text {,loc }}^{\infty}$ with $\beta^{\prime}<\beta=\min (\alpha, 4)$. By iteration, we can obtain the result for any $\alpha$. Since the above proof is local, it works for any $S$.

\section{APPENDIX}

Some properties of $L^{p}$-Sobolev spaces. In this appendix, we provide, for the reader's convenience, a summary of various facts about $L^{p}$-Sobolev spaces used in this paper. $L_{\alpha}^{p}$ is defined to be the set of functions, $f$, in $L^{p}$ so that there exists $g \in L^{p}$ with $\hat{f}(k)=\left(1+k^{2}\right)^{\alpha / 2} \hat{g}(k)$. If $\beta$ is a 
multi-index with $|\beta|<\alpha$, then $k^{\beta}\left(1+k^{2}\right)^{-\alpha / 2}$ is the Fourier transform of a function in $L^{1}$, so since convolution with $L^{1}$ functions leaves $L^{p}$ invariant, we see that if $\alpha<\gamma$, then $L_{\alpha}^{p} \subset L_{\gamma}^{p}$, and if $\alpha>n$ is an integer, all $D^{\beta} f \in L^{p}$ for $|\beta| \leq n$. Only for $n=1$ will we require the more subtle fact [13] that this holds if $\alpha=n$ when $p \neq 1, \infty$ (see Theorem A.3). In particular, it is obvious that if $\alpha=2 l$ is an even integer, then $f \in L_{\alpha}^{p}$ if and only if $f, \Delta f, \ldots, \Delta^{k} f \in L^{p}$.

We will exploit the theory of complex interpolation [1] for the $L_{\alpha}^{p}$ 's. The key fact [1] is that if $1<p_{0}, p_{1}<\infty$, then $\left(L_{\alpha_{0}}^{P_{0}}, L_{\alpha_{1}}^{p_{1}}\right)_{\theta}=L_{\alpha_{\theta}}^{p_{\theta}}$ where $p_{\theta}^{-1}=\theta p_{1}^{-1}+(1-\theta) p_{0}^{-1}$ and $\alpha_{\theta}=\theta \alpha_{1}+(1-\theta) \alpha_{0}$.

We will occasionally state the results in a less general form than that which is valid if the proof of the less general result is easier, and the less general result is all that is needed in the text.

THEOREM A.1. Let $f$ be $C^{2 k}$ for $k$, an integer. Then the map $M_{f}$ : $g \mapsto$ fg maps $L_{\alpha}^{p}$ to itself for all $p \neq 1, \infty$ and all $\alpha \in[0,2 k]$.

Proof. By interpolation, we need only consider the cases $\alpha=0,2 k$, $\alpha=0$ is trivial. For $\alpha=2 k$, note that $\Delta^{k}(f g)=f\left(\Delta^{k} g\right)+g\left(\Delta^{k} f\right)+R$ where $R$ involves products of derivative of degree $\beta<2 k$. Since $g \in L_{2 k}^{p}$, $D^{\beta} g \in L^{p}$ for all $\beta<2 k$ and $\Delta^{k} g \in L^{p}$, the result follows.

In particular, this result shows that $L_{\alpha}^{p} \subset L_{\alpha, \text { loc }}^{p}$. Let $L_{\alpha, \text { comp }}^{p}$ denote the $f \in L_{\alpha}^{p}$ with compact support. The $g$ with $\hat{g}=\left(1+k^{2}\right)^{\alpha / 2} \hat{f}$ will not have compact support if $\alpha \neq 2 l$, but since the Fourier transform of $\left(1+k^{2}\right)^{\alpha / 2}$ is a distribution given away from zero by a function with exponential decay, $|g(x)| \leq C e^{-D|x|}$ for $x<p$. Thus: $L_{\alpha, \text { loc }}^{q}$.

THEOREM A.2. If $1 \leq q<p \leq \infty, L_{\alpha, \mathrm{comp}}^{p} \subset L_{\alpha, \mathrm{comp}}^{q}$ and $L_{\alpha, \mathrm{loc}}^{p} \subset$

The next few results require a basic fact about $L_{\alpha}^{p}$ proven, for example, in Stein [13], p. 135ff.

Theorem A.3. Let $1<p<\infty$. Then $f \in L_{1}^{p}$ if and only if $f$ and $\vec{\nabla} f$ (distributional derivative) lie in $L^{p}$ and $\|f\|_{L_{\alpha}^{p}}$ is equivalent to $\|f\|_{p}+\|\vec{\nabla}\|_{p}$.

There is a more general $L_{\alpha}^{p}$-Hölder inequality: $L_{\alpha}^{p} \cdot L_{\alpha}^{q}$ lies in $L_{\alpha}^{r}$ $\left(r^{-1}=p^{-1}+q^{-1}\right)$. We only require the result that follows:

Theorem A.4. If $f \in L_{1}^{p}$ and $g \in L_{\beta}^{q}$ with $0 \leq \beta \leq 1$ and $p(p-1)^{-1}$ $<q<\infty$, then $f g \in L_{\beta}^{r}$ with $r^{-1}=p^{-1}+q^{-1}$. 
Proof. By interpolation, we need only prove the result for $\beta=0,1$. $\beta=0$ is the ordinary Hölder inequality. $\beta=1$ follows from Theorem A.3 and the ordinary Hölder inequality if we note $\nabla(f g)=(\nabla f) g+f(\nabla g) \cdot \square$

THEOREM A.5. If $f$ is a continuous function on $R^{\nu}$ everywhere strictly positive, and $f \in L_{1, \text { loc }}^{p}$, then $f^{-1} \in L_{1, \mathrm{loc}}^{p}$.

Proof. By mollifying $f$, it is easy to check that, in distributional sense, $\nabla\left(f^{-1}\right)=-f^{-2} \nabla f$. Thus $\nabla f$ in $L_{\text {loc }}^{p}$ and $f^{-1}$ in $L_{\text {loc }}^{\infty}$ implies that $\nabla f^{-1} \in L_{\text {loc }}^{p}$.

The following result is a special case of the Gagliardo-Nirenberg inequalities (see e.g. [9]).

TheOREM A.6. If $f \in L^{\infty} \cap L_{2, \mathrm{loc}}^{2}$, then $\nabla f \in L_{\mathrm{loc}}^{4}$ and so $f \in L_{1, \mathrm{loc}}^{4}$.

Next we will construct $f \in L^{\infty}$ with compact support so that $f$ lies in no $L_{\alpha}^{1}(\alpha>0)$ (and so by Theorem A.2 in no $L_{\alpha}^{p}(\alpha>0)$ ).

THEOREM A.7. There exists $f$, a continuous function, with compact support, so $f \notin \bigcup_{\alpha>0} L_{\alpha}^{1}$.

Proof. Let $k_{n}=\left(2^{n}, 0, \ldots, 0\right) \in R^{\nu}$, let $g \in C_{0}^{\infty}\left(R^{\nu}\right)$ with $\hat{g}(0)=1$ and let $f(x)=\sum_{n} n^{-2} e^{i k_{n} \cdot x} g(x)$. The sum converges uniformly, so $f$ is continuous. Moreover, since $\hat{g}(k)$ decays faster than $k^{-1}$, it is easy to see that $n^{2} \hat{f}\left(k_{n}\right) \rightarrow 1$. In particular, $\left(1+\left|k_{n}\right|^{2}\right)^{\alpha / 2} \hat{f}\left(k_{n}\right) \rightarrow \infty$ for any $\alpha>0$. It follows that $f \notin L_{\alpha}^{1}$ for any $\alpha>0$.

As a final result, we need

THeOREM A.8. If $f, \Delta f \in L_{\alpha, \text { loc }}^{p}$, then $f \in L_{\alpha+2, \mathrm{loc}}^{p}$.

Proof. The proof is only somewhat involved since we have the loc's. For $\varphi \in C_{0}^{\infty}$,

$$
(1-\Delta)(\varphi f)=g-2 \nabla \cdot(\nabla \varphi f)
$$

where $g=\varphi(1-\Delta) f+(\Delta \varphi) f \in L_{\alpha}^{p}$ by hypothesis. Thus

$$
(1-\Delta)^{1 / 3}(\varphi f)=h-\sum_{i} A_{i}\left[\left(\nabla_{\imath} \varphi\right) f\right]
$$


where $h=(1-\Delta)^{-2 / 3} g \in L_{\alpha+4 / 3}^{p}$ and $A_{i}=(1-\Delta)^{-3 / 4} \nabla_{i}$ is convolution with a function in $L^{1}$ and so a bounded map on each $L_{\alpha}^{p}$. By 91) and $f \in L_{\alpha, \mathrm{loc}}^{p}$, we conclude that $(1-\Delta)^{1 / 3}(\varphi f) \in L_{\alpha}^{p}$, so $f \in L_{\alpha+2 / 3, \mathrm{loc}}^{p}$. Iterating this argument twice, we find $f \in L_{\text {loc }}^{p}$. The iteration stops because $h$ is only in $L_{\alpha+4 / 3}^{p}$.

\section{REFERENCES}

[1] A. Calderon, Intermediate spaces and interpolation, Studia Math., Spec. Series, 1 (1963), 31-190.

[2] R. Carmona, Regularity properties of Schrödinger and Dirichlet semigroups, J. Func. Anal., 17 (1974), 227-237.

[3] I. Herbst and A. Sloan, Perturbations of translation invariant positivity preserving semigroups in $L^{2}(R)$, Trans. Amer. Math. Soc., 236 (1978), 325-360.

[4] W. Hunziker, Space-time behavior of Schrödinger wave functions, J. Math. Phys., 7 (1966), 300-304.

[5] T. Kato, Schrödinger operators with singular potentials, Israel J. Math., 13 (1973), 135-148.

[6] _ Fundamental properties of Hamiltonian operators of Schrödinger type, Trans. Amer. Math. Soc., 70 (151), 195-211.

[7] M. Kon, Problem \#6 in Problem List in Partial Differential Operators, Notices Amer. Math. Soc., 31 (184), 631.

[8] V. Kovalenko and $\mathrm{Yu}$. Semenov, Some problems on expansions in generalized eigenfunctions of the Schrödinger operator with strongly singular potentials, Russian Math. Surveys, 33 (1978), 119-157.

[9] H. Leinfelder and C. Simader, Schrödinger operators with singular magnetic vector potentials, Math. Z., 176 (1981), 1-19.

[10] C. Radin and B. Simon, Invariant domains for the time-dependent Schrödinger equation., J. Differential Equations, 29 (1978), 289-296.

[11] B. Simon, Functional Integration and Quantum Physics, Academic Press, New York, 1979.

[12] _ Schrödinger semigroups, Bull. Amer. Math. Soc., 7 (1982), 447-526.

[13] E. Stein, Singular Integrals and Differentiability Properties of Functions, Princeton University Press, 1970.

Received December 17, 1984 and in revised form March 26, 1985. Research suported by USNSF under grant MCS-81-20833.

CALIFornia Institute of TeChNology

PASADENa, CA 91125 


\section{PACIFIC JOURNAL OF MATHEMATICS EDITORS}

\author{
V. S. VARAdarajan (Managing Editor) \\ University of California \\ Los Angeles, CA 90024 \\ Hebert Clemens \\ University of Utah \\ Salt Lake City, UT 84112 \\ Charles R. DePrima \\ California Institute of Technology \\ Pasadena, CA 91125
}

R. FINN

Stanford University

Stanford, CA 94305

HeRManN FLASChKa

University of Arizona

Tucson, AZ 85721

RAMESH A. GANGOlli

University of Washington

Seattle, WA 98195

ROBION KIRBY

University of California

Berkeley, CA 94720

\author{
C. C. MOORE \\ University of California \\ Berkeley, CA 94720 \\ H. SAMELSON \\ Stanford University \\ Stanford, CA 94305 \\ HAROLD STARK \\ University of California, San Diego \\ La Jolla, CA 92093
}

\section{ASSOCIATE EDITORS}

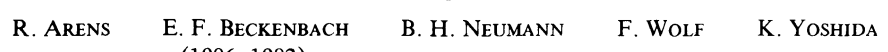

(1906-1982)

\section{SUPPORTING INSTITUTIONS}

\begin{abstract}
UNIVERSITY OF ARIZONA
UNIVERSITY OF BRITISH COLUMBIA

CALIFORNIA INSTITUTE OF TECHNOLOGY

UNIVERSITY OF CALIFORNIA

MONTANA STATE UNIVERSITY

UNIVERSITY OF NEVADA, RENO

NEW MEXICO STATE UNIVERSITY

OREGON STATE UNIVERSITY
\end{abstract}

\author{
UNIVERSITY OF OREGON \\ UNIVERSITY OF SOUTHERN CALIFORNIA \\ STANFORD UNIVERSITY \\ UNIVERSITY OF HAWAII \\ UNIVERSITY OF TOKYO \\ UNIVERSITY OF UTAH \\ WASHINGTON STATE UNIVERSITY \\ UNIVERSITY OF WASHINGTON
}

The Supporting Institutions listed above contribute to the cost of publication of this Journal, but they are not owners or publishers and have no responsibility for its content or policies.

Mathematical papers intended for publication in the Pacific Journal of Mathematics should be in typed form or offset-reproduced (not dittoed), double spaced with large margins. Please do not use built up fractions in the text of the manuscript. However, you may use them in the displayed equations. Underline Greek letters in red, German in green, and script in blue. The first paragraph must be capable of being used separately as a synopsis of the entire paper. In particular it should contain no bibliographic references. Please propose a heading for the odd numbered pages of less than 35 characters. Manuscripts, in triplicate, may be sent to any one of the editors. Please classify according to the scheme of Math. Reviews, Index to Vol. 39. Supply name and address of author to whom proofs should be sent. All other communications should be addressed to the managing editor, or Elaine Barth, University of California, Los Angeles, California 90024.

There are page-charges associated with articles appearing in the Pacific Journal of Mathematics. These charges are expected to be paid by the author's University, Government Agency or Company. If the author or authors do not have access to such Institutional support these charges are waived. Single authors will receive 50 free reprints; joint authors will receive a total of 100 free reprints. Additional copies may be obtained at cost in multiples of 50 .

The Pacific Journal of Mathematics is issued monthly as of January 1966. Regular subscription rate: $\$ 190.00$ a year (5 Vols., 10 issues). Special rate: $\$ 95.00$ a year to individual members of supporting institutions.

Subscriptions, orders for numbers issued in the last three calendar years, and changes of address should be sent to Pacific Journal of Mathematics, P.O. Box 969, Carmel Valley, CA 93924, U.S.A. Old back numbers obtainable from Kraus Periodicals Co., Route 100, Millwood, NY 10546.

The Pacific Journal of Mathematics at P.O. Box 969, Carmel Valley, CA 93924 (ISSN 0030-8730) publishes 5 volumes per year. Application to mail at Second-class postage rates is pending at Carmel Valley, California, and additional mailing offices. Postmaster: Send address changes to Pacific Journal of Mathematics, P.O. Box 969, Carmel Valley, CA 93924.

PUBLISHED BY PACIFIC JOURNAL OF MATHEMATICS, A NON-PROFIT CORPORATION

Copyright $\odot 1986$ by Pacific Journal of Mathematics 


\section{Pacific Journal of Mathematics}

\section{Vol. 122, No. 2 \\ February, 1986}

Gideon Amit and David Chillag, On a question of Feit concerning character values of finite solvable groups ......................257

Constantin Gelu Apostol and Frank Larkin Gilfeather, Isomorphisms modulo the compact operators of nest algebras ................263

Parviz Azimi and James Neil Hagler, Examples of hereditarily $l^{1}$ Banach

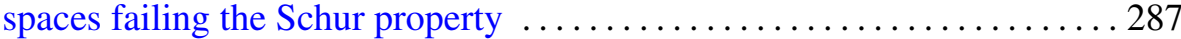

Brian Evan Blank, Boundary behavior of limits of discrete series representations of real rank one semisimple groups . . . . . . . . . . 299

Jeffrey Carroll, Some undecidability results for lattices in recursion theory

Gerald Howard Cliff and Alfred Rheinhold Weiss, Crossed product and

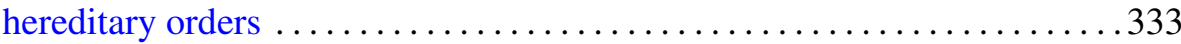

Ralph Cohen, Realizing transfer maps for ramified coverings . . . . . . . . 347

Ronald James Evans, Hermite character sums . .................. 357

C. L. Frenzen and Roderick Sue-Chuen Wong, Asymptotic expansions of the Lebesgue constants for Jacobi series . . . . . . . . . . . . . . . . 391

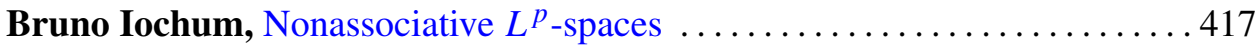

John McDonald, Unimodular approximation in function algebras ....... 435

John Robert Quine, Jr., Ramification and unintegrated value distribution . . 441

Marc Raphael, Commutants of quasisimilar subnormal operators ........ 449

Parameswaran Sankaran and Peter Zvengrowski, On stable

parallelizability of flag manifolds

Helga Schirmer, A relative Nielsen number

Barry Simon, Schrödinger semigroups on the scale of Sobolev spaces . . . . . 475

Viakalathur Shankar Sunder, Stochastic integration in Fock space

Jan de Vries, A note on the $G$-space version of Glicksberg's theorem 